Relations industrielles

Industrial Relations

\title{
La seconde guerre mondiale, le Conseil supérieur du travail et les lois ouvrières de 1944 (1940-1945)
}

\section{Roger Chartier}

Volume 18, numéro 3, juillet 1963

URI : https://id.erudit.org/iderudit/1021400ar

DOI : https://doi.org/10.7202/1021400ar

Aller au sommaire du numéro

Éditeur(s)

Département des relations industrielles de l’Université Laval

ISSN

0034-379X (imprimé)

1703-8138 (numérique)

Découvrir la revue

Citer cet article

Chartier, R. (1963). La seconde guerre mondiale, le Conseil supérieur du travail et les lois ouvrières de 1944 (1940-1945). Relations industrielles / Industrial Relations, 18(3), 346-362. https://doi.org/10.7202/1021400ar
Résumé de l'article

Voici le septième d'une série de textes sur la législation québécoise du travail (et sur les services connexes du Ministère du travail) de 1885 à nos jours. Ces notes pourront un jour servir à une histoire plus méthodique des relations du travail au Québec.
Tous droits réservés (C Département des relations industrielles de l’Université Laval, 1963
Ce document est protégé par la loi sur le droit d'auteur. L'utilisation des services d'Érudit (y compris la reproduction) est assujettie à sa politique d'utilisation que vous pouvez consulter en ligne.

https://apropos.erudit.org/fr/usagers/politique-dutilisation/ 
Contribution à l'histoire de la législation

québecoise du travail: VII

\section{La seconde guerre mondiale, \\ le Conseil supérieur du travail et les lois ouvrièresde 1944 (1940-1945)}

\section{Roger Chartier}

Voici le septième d'une série de textes sur la législation québecoise du travail (et sur les services connexes du Ministère du travail) de 1885 à nos jours. Ces notes pourront un jour servir à une histoire plus méthodique des relations du travail au Québec.

La période qui s'ouvre ici en est une d'activité économique intense. La guerre à ses débuts aura tôt fait d'entraîner l'industrie de la province et du pays dans un rythme extrêmement rapide, un rythme factice si l'on veut, mais qui, une fois atteint, se maintiendra jusqu'à nos jours en dépit de tous les pronostics. L'énorme machine à faire la guerre prendra bien quelques mois à se mettre en branle. Le passage d'une économie de paix au ralenti à une économie de guerre à outrance ne se fera pas sans heurts et sans atermoiements. A la production d'armes, de munitions, de vêtements, de vivres, il faut habituer une main-d'oeuvre en grande partie nouvelle. Près d'un demi-million de femmes envahissent le marché du travail. L'état de crise déplace la juridiction et les pouvoirs en matières sociales et industrielles du côté des autorités fédérales. La Province de Québec, par son Ministère du travail, offrira l'exemple d'une parfaite collaboration en ces domaines.

CHARTIER, Roger, M.Sc.soc., professeur au Département des relations industrielles de la Faculté des sciences sociales de l'Université Laval et directeur général du personnel de la Commission hydroélectrique de Québec, études de doctorat en sociologie à l'Université de Chicago.

(*) Cette série d'articles est la synthèse d'une \& Etude analytique et évolutive des services du Ministère du travail et de la législation ouvrière et sociale de la province de Québec, 1885-1952 》 préparée par l'auteur à la demande du Ministère, et reproduite ici avec sa permission. 
Nous avons vu antérieurement que le régime d'assistance-chômage instauré par la Loi fédérale de 1930 pour remédier au chômage, continué sous diverses formes pendant toute la durée de la crise - de concert avec les provinces, - prit fin le 31 mars 1941. Alors seulement le chômage avait pratiquement cessé en raison de l'activité économique du temps de guerre: on sait que l'effort industriel du Canada au cours de la dernière guerre dépassa toutes les prévisions, surtout dans le domaine des armements.

La Loi des mesures de guerre de 1914, qui revint automatiquement en vigueur au début de la seconde guerre mondiale, autorisait le gouvernement fédéral à prendre toutes les mesures jugées nécessaires pour la sécurité, l'ordre et le bien-être du pays. Dès le début de la guerre, la Loi fédérale des enquêtes en matière de différends industriels - ancienne «Loi Lemieux》 de 1907, modifiée en 1925 et adoptée par la Législature provinciale en 1932, - qui interdisait tout arrêt de travail dans les services publics avant la tenue d'une enquête conciliatrice, s'appliqua également aux industries de guerre (C.P. 3495). En juin 1940, l'arrêté fédéral C.P. 2685 appuyait le principe de la liberté syndicale et encourageait la signature de conventions collectives, enjoignant aux parties de ne pas suspendre autrement que par conventions les conditions de travail établies. Le gouvernement fédéral définissait en 1940 également sa politique sur les salaires en temps de guerre, pour la gouverne des commissions de conciliation et d'enquête: le C.P. 7440 plafonnait les salaires au niveau le plus élevé depuis 1926, encourageant le paiement d'une indemnité de vie chère correspondant à la hausse du coût de la vie. La plupart de ces arrêtés n'avaient rien de coercitif, mais leur influence créa peu à peu une «coutume» industrielle.

La Loi fédérale de l'assurance-chômage une fois en vigueur (1940), la Commission d'assurance-chômage se chargea des bureaux de placement dans tout le pays, comme le stipulait la loi. Son rôle en était un de coordination efficace plutôt que de création. Dans le cas du Québec en particulier, elle profitera au maximum de la solide organisation du service provincial de placement, dont elle conservera en grande partie la structure et dont elle continuera d'employer le personnel.

\section{L'ANNÉE 1940 AU PROVINCIAL}

Dans le domaine législatif, l'année 1940 est une année fort active. Le Parlement provincial vote d'abord la Loi instituant le Conseil supérieur du Travail (4 Geo. VI, ch. 37). Le Conseil ainsi créé est un organisme consultatif chargé d'étudier les questions sociales: protection des ouvriers, rationalisation du travail, convention collective, salaire minimum, inspection du travail, prévention des accidents du travail, apprentissage, orientation professionnelle, réhabilitation des chômeurs, assurance et assistance sociales, suppression des taudis et accession des travailleurs à la propriété. Le nouvel organisme sera formé de $24 \mathrm{mem}-$ 
bres désignés par le lieutenant-gouverneur en conseil sur la recommandation du ministre du Travail: 8 de ces membres représenteront le Capital, 8 le Travail; 8 autres seront économistes ou sociologues; 6 membres additionnels - 3 du Ministère du travail et 3 du Ministère du commerce et de l'industrie - pourront s'adjoindre au groupe initial. Les membres réguliers, en fonction durant 3 ans, seront rééligibles, et ne recevront aucune rémunération. Le Conseil élira un président et deux vice-présidents, ainsi qu'une Commission permanente de six membres -2 du Capital, 2 du Travail et 2 économistes-sociologues, plus deux membres adjoints représentant les deux ministères précités; les membres réguliers sont élus pour un an. La Commission a pour fonction de préparer les travaux du Conseil et de représenter celui-ci auprès du ministre. Le secrétaire de la Commission joue le même rôle auprès du Conseil. Le Conseil supérieur du Travail jouera un rôle souvent effacé, mais très important dans l'évolution de notre législation sociale.

La même année, la Loi de la convention collective abroge et remplace, en la modifiant, la Loi relative aux salaires des ouvriers de 1937. C'est cette loi (4 Geo. VI, ch. 38) qui, avec ses amendements, nous régit encore aujourd'hui, dans le domaine de l'extension juridique des contrats collectifs. Le nouveau texte est le fruit du travail de trois juristes. Des considérations initiales servent à mieux définir l'esprit de la Loi, qui n’a pas changé:

Crnsidérant que la justice sociale impose la réglementation du travail lorsque la situation économique entraîne pour le salarié des conditions contraires à l'équité;

Considérant que tolérer l'acceptation forcée d'une rémunération insuffisante c'est ne pas tenir compte de la dignité du travail et des besoins du salarié et de sa famille;

Considérant qu'il est opportun d'adopter, d'étendre et de rendre obligatoires les conditions de travail consignées dans les conventions collectives, tant pour prévenir la concurrence déloyale faite aux signataires que pour établir le juste salaire et satisfaire à l'équité...

Le nouveau texte précise que l'on peut avoir quatre types de décret: «rendant obligatoire, modifiant, prolongeant ou abrogeant la convention collective». On définit que l' « exploitation agricole est une ferme mise en valeur par l'exploitant lui-même ou par l'entremise d'employés ». La rémunération doit être «en monnaie courante ». « Le décret peut ordonner que certaines personnes ou associations soient traitées comme parties contractantes » (article 10, 2e paragraphe). Le lieutenant-gouverneur en conseil peut prolonger - non plus modifier ou en tout temps révoquer le décret. Toute rétroactivité est éliminée à partir de la date de la sanction - dans l'ancienne loi, il y avait un jeu de 4 mois. La nouvelle loi pourvoit pour la première fois à la nomina- 
tion de substituts au comité paritaire. Celui-ci aura désormais 15 jours - non plus 30 - pour poursuivre en recouvrement au nom du salarié lésé; il pourra reprendre l'instance au nom du salarié négligent, et régler par compromis en cas de réclamations de salaire; l'administrateur de ses fonds devra fournir un cautionnement; le comité pourra exiger l'affichage des documents nécessaires à l'application du décret, ainsi qu'un système d'enregistrement, un rapport mensuel de l'employeur, et un certificat de classification pour les employés non assujettis à l'obligation de détenir un certificat de qualification. Celui-ci peut être rendu obligatoire dans toute la juridiction territoriale du décret - auparavant, il ne pouvait l'être que dans les villes de plus de 5,000 âmes; les employés de bureau et les journaliers peuvent en être exemptés. Voilà autant de modifications qu'apporte le nouveau texte. Il est à noter que le contrôle fédéral des salaires en temps de guerre, soit de 1941 à 1946, eut préséance sur la juridiction provinciale et paralysa pour un temps le développement de l'extension juridique des conventions collectives.

C'est en 1940 encore qu'est adoptée une nouvelle Loi du salaire minimum (4 Geo. VI, ch. 39), qui abroge et remplace la Loi des salaires raisonnables de 1937 . Un «considérant» indique bien le caractère de complémentarité de cette loi par rapport à la précédente:

Considérant que s'il est préférable que la réglementation nécessaire soit faite par le moyen de conventions collectives rendues obligatoires, il faut cependant, lorsque cette méthode n'est pas applicable, prévoir la fixation, par une corporation publique, des minima de salaires requis pour empêcher les abus...

La nouvelle loi s'applique à «tous les salariés 》, hommes et femmes, dont le travail se fait dans la province ailleurs que dans l'agriculture et le service domestique, et qui ne sont pas déjà régis par une convention ou un décret plus avantageux. Pour la faire respecter, une corporation est établie: c'est la Commission du salaire minimum, composée d'au plus cinq membres, qui remplace l'Office des salaires raisonnables, avec sensiblement les mêmes pouvoirs. Les comités de conciliation disparaissent. L'article 13 élargit le cadre des décisions de la Commission: «La Commission peut, par ordonnance, déterminer, pour des périodes de temps et des territoires désignés, le taux du salaire minimum payable à toute catégorie de salariés qu'elle indique, les termes de paiement, la durée du travail, les conditions de l'apprentissage, le rapport entre le nombre d'ouvriers qualifiés et celui des apprentis dans une entreprise donnée, la classification des opérations et les autres conditions de travail jugées conformes à l'esprit de la loi ». Les ordonnances émises, tout comme les décrets, sont d'ordre public; tout employeur visé doit s'y soumettre sous peine de sanction. Les ordonnances et règlements en cours demeurent en vigueur jusqu'à la date de leur expiration.

En 1940 toujours, la Loi des pensions de vieillesse est modifiée de façon à ne profiter qu'à ceux qui sont vraiment dans le besoin (ch. 42): 
le réclamant doit se présenter à l'inspecteur avec une formule signée par le secrétaire de sa municipalité et indiquant les immeubles qui ont été inscrits à son nom ou au nom de son conjoint durant les cinq années précédentes. La même précaution s'applique pour l'aide aux aveugles et aux mères nécessiteuses. Cette dernière loi est modifiée (ch. 43) quant aux conditions auxquelles la mère sera bénéficiaire: il lui suffira désormais de n'avoir qu'un enfant au lieu de deux; elle doit être «veuve ou épouse d'un mari absent depuis cinq ans ou totalement invalide et hospitalisé; sujet britannique depuis au moins quinze ans ou à sa naissance; domiciliée dans la province depuis sept ans et y ayant effectivement résidé au moins 1825 jours...»

Sur le plan administratif, l'activité des divers services du Ministère du travail en 1940 est en tous points remarquable. De 39 qu'il était en 1939 , le nombre des décrets en vigueur passe à 103 en 1940. On compte 13 décrets ( 21 zones) chez les barbiers-coiffeurs et 11 autres ( 25 zones) dans la construction. Les salaires s'accroissent d'environ $10 \%$. Le premier rapport de la nouvelle Commission du salaire minimum, présidée par le juge Ferdinand Roy et comptant un personnel de 122 à Québec, Montréal, Sherbrooke, Hull et Trois-Rivières, nous apprend que les ordonnances 1,2 et 3 régissant le travail féminin dans les régions rurales ont été abrogées, l'ordonnance 4 - réglementant le travail dans les cités et villes - demeurant la seule ordonnance générale. Cette ordonnance divise la province en 4 zones, et uniformise les conditions pour les cités et villes rurales ou urbaines. Elle abroge l'arrêté du 22 décembre 1938 qui exemptait les corporations municipales et scolaires. La nouvelle loi supprime le pouvoir qu'avait l'Office des salaires raisonnables d'émettre des règles interprétatives incorporées aux ordonnances. La Commission doit dorénavant veiller à l'application des conventions visées par la Loi des syndicats professionnels. Aux 17 ordonnances spéciales en vigueur le ler juillet 1939, la Commission en ajoute 12. Par son règlement B-1 amendé, approuvé le 30 novembre 1939, l'Office avait ordonné un prélèvement de $1 / 3$ de $1 \%$ sur les salaires soumis; depuis le ler janvier 1940 , on a ainsi perçu $\$ 220,438.49$.

L'officier des justes salaires continue à faire respecter la loi dans 144 contrats de voirie et de travaux publics, dont la liste est fournie au Ministère du travail. L'officier distribue des cartes d'emploi - 5,000 à Montréal seulement - pour connaître la nature du travail accompli par chaque ouvrier et déterminer ainsi la rémunération à laquelle il a droit. - Les statistiques du service de conciliation et d'arbitrage se font plus précises. Le service est intervenu dans 19 conflits, dont 15 ont dégénéré en grèves, la plupart à la suite du refus de l'employeur de reconnaître le syndicat et de négocier avec lui. L'activité de grève est forte dans le textile et le vêtement. En tout, près de 10,000 travailleurs sont impliqués, qui perdent plus de 100,000 journées de travail. - Chez les inspecteurs des établissements industriels, commerciaux et publics, on compte un personnel de 53, sous la direction du nouveau chef Clovis 
Bernier. Le rapport de 1940 rend compte d'une réorganisation du personnel et des dossiers. - Pour les machines fixes et les appareils sous pression, on a 43 employés; au cours de l'année se crée le sous-service de réfrigération, devenu nécessaire à cause du nombre toujours croissant des appareils frigorifiques et de climatisation. - Rien de nouveau chez les électriciens et chez les mécaniciens en tuyauterie. - 93 employés voient au placement de 84,119 sans-travail (sur 215,000 inscriptions), dont 39,123 femmes. Les placements féminins augmentent de $24 \%$ sur l'année précédente, tandis que les placements masculins baissent de $16 \%$. Les bureaux de Bagotville, de Maisonneuve et de St-Henri pour les hommes, sont fermés. - La comptabilité du ministère révèle un revenu de $\$ 373,591.53$, et des dépenses de $\$ 6,124,358.78$, accrues considérablement par l'application des trois lois d'assistance sociale.

\section{L'ANNÉE 1941}

L'arrêté fédéral C.P. 8253 du 24 octobre 1941, qui remplace le C.P. 7440 sur les salaires en temps de guerre et sur l'indemnité de vie chère, étend le contrôle des salaires à tous les employeurs: les taux sont établis au niveau du 15 novembre 1941. L'arrêté établit un rouage permanent pour sa mise en vigueur, le Conseil national du travail en temps de guerre qui, avec l'aide de neuf conseils régionaux, contrôlera l'application de l'arrêté et de la politique des justes salaires du gouvernement.

Dans le domaine de l'extension juridique, 225 arrêtés sont pris en exécution de la loi; 26 nouveaux décrets sont sanctionnés, ce qui porte à 97 le nombre des décrets en vigueur au cours de l'année (de neuf mois, car l'exercice financier se terminera désormais le 31 mars au lieu du 30 juin). - Le personnel chargé d'appliquer la Loi du salaire minimum passe de 122 à 171 au cours des neuf mois. Le taux du prélèvement est abaissé de $1 / 3$ à $1 / 5$ de $1 \%$. Les recouvrements de salaires se chiffrent à $\$ 79,000$. La Commission accuse un surplus de $\$ 565,695.03$, qui sert à combler le déficit de $\$ 411,590.42$ accumulé au 30 juin 1940 . - L'inspection du travail compte 4 employés de plus (57); les inspecteurs notent une activité industrielle intense, surtout dans le domaine des munitions et des approvisionnements, et la présence d'un grand nombre de jeunes gens de 16 à 18 ans, de jeunes filles et de femmes; les visites de toutes sortes se multiplient dans les établissements industriels et commerciaux et dans les édifices publics. - Le service de placement compte 21 bureaux réguliers, 6 temporaires et 102 employés. En neuf mois, les placements effectués (117,598, dont 38,429 femmes) dépassent de $40 \%$ ceux de l'année précédente. Le bâtiment vient au premier rang avec 50,422 cas; puis suivent l'industrie forestière, les travaux d'occasion et le service domestique. - La Commission des accidents du travail, dans son 3e rapport annuel - au 31 décembre $1940-$, indique que le fonds d'accident a un surplus de $\$ 1,228,938.69$, après réduction rétroactive de taux d'environ $\$ 500,000$ pour 1940 . Notons que les surplus avaient été de $\$ 314,826$ de 1931 à 1938 , de $\$ 646,402$ en 1939 et de $\$ 267,709$ en 1940 . Le bond est donc assez remarquable. Les réclamations se chiffraient à 327,487 de 1931 à $1938,53,942$ en 1939 et 65,704 
en 1940. L'administration au cours des neuf mois a coûté $\$ 468,357.13$, y compris les frais légaux et les octrois de prévention. A l'application des trois lois d'assistance sociale - pensions de vieillesse, pensions aux aveugles, allocations aux mères nécessiteuses - 73 employés de bureau et 111 inspecteurs s'affairent. Au 31 décembre 1940, 69,487 pensions de vieillesse ont été payées, la province seule $(25 \%)$ dépensant plus de $\$ 10 \mathrm{r} / 2$ millions. A la même date, 2,205 pensions au coût de près de $\$ 300,000$ pour la province $(25 \%)$ ont été versées aux aveugles. Enfin, 7,074 allocations à des mères nécessiteuses ont coûté plus de $\$ 4$ millions à la province. - Les revenus du ministère se chiffrent à $\$ 340,088.30$, et les dépenses à \$4,083,350.73.

En cette année 1941, trois amendements méritent d'attirer notre attention. Le premier (5 Geo. VI, ch. 59) modifie la Loi des syndicats professionnels, en déterminant que la constitution du syndicat devra prévoir au moins une assemblée générale par trimestre, faire un devoir aux officiers, délégués et chargés d'affaires de présenter au moins un rapport annuel, imposer à chaque membre le paiement d'une cotisation d'au moins un dollar sous peine d'expulsion, et enfin statuer que le procès verbal de chaque assemblée doit être approuvé au plus tard à la séance suivante. L'amendement précise encore que «la réduction à moins de vingt du nombre des membres en règle du syndicat en entraîne de plein droit la dissolution ». - Un deuxième amendement (ch. 60) ajoute à la Loi de la convention collective les paragraphes suivants: «Le décret peut rendre obligatoire des prix minima à être chargés au public pour les services des barbiers et coiffeurs » - c'était là faire une exception à la règle qui veut que la législation du travail s'applique aux rapports naissant de la production et de la distribution des biens et services, et non pas à leur consommation; " au cas de poursuite sommaire, il y a droit d'appel quand l'amende prévue pour l'infraction est de deux cents dollars ou plus »; «le comité paritaire peut accorder à ses membres, outre les frais de déplacement, des jetons de présence n'excédant pas $\$ 5$ chacun, pour pas plus d'une assemblée par semaine ». - Un troisième amendement modifie la Loi de l'asistance aux mères nécessiteuses, en incluant comme bénéficiaire (ch. 53) l'épouse ou la veuve d'un sujet britannique et en excluant l'épouse d'un mari hospitalisé.

Les années 1942 et 1943 sont peu remarquables pour la Province au point de vue législatif, dans les domaines qui nous occupent. ${ }^{1}$ Dans son rapport de 1942, le sous-ministre Tremblay nous apprend que:

Malgré la mise en vigueur, au 15 novembre 1941, de l'arrêté fédéral C.P. 8253 , les conventions collectives approuvées par décret en vertu de la Loi de la convention collective, de même que les ordonnances de la Commission du salaire minimum, sont demeurées en vigueur.

(1) Mentionnons toutefois l'adoption d'une loi nouvelle, un peu plus élaborée, des mécaniciens de machines fixes ( $6 \mathrm{Geo}$. VI, ch. 52, 1942), et d'amendements mineurs à toutes les lois de sécurité. 
Ces décrets et ordonnances, quant aux taux de salaires et autres conditions affectant la rémunération qui y sont contenus, sont demeurés inchangés. Si des modifications y ont été apportées, ce n'est évidemment qu'après l'obtention, au préalable, de l'autorisation du Conseil national ou du Conseil régional du travail en temps de guerre.

(p. 6)

Les services nécessaires à l'application de la Loi de la convention collective et de la Loi du salaire minimum demeurent intacts. A la fin de l'exercice financier 1941-42, 87 décrets sont en vigueur, dont quatre nouveaux; à la fin de 1942-43, nous en comptons 99, dont 45 contiennent une clause d'indemnité de vie chère. Les comités paritaires collaborent étroitement avec les autorités fédérales pour la mise à exécution de la politique canadienne de stabilisation des salaires; tous deviennent des agences officielles du Conseil régional du travail pour l'application des arrêtés fédéraux C.P. 7679 - sur les salaires minima dans les industries de guerre - et C.P. 8253; de plus, 175 inspecteurs de comités paritaires sont individuellement agents du fédéral à cette fin. Soixante-dix comités prélèvent des cotisations, dont 67 le maximum, soit $1 / 2 \mathrm{de} 1 \%$. Les décrets en vigueur couvrent en 1942, 35 industries, commerces ou occupations, 9,634 employeurs, 86,965 hommes et 29,014 femmes. M. Cyprien Miron, chef du service de conciliation, est en même temps inspecteur en chef des comités paritaires.

La Commission du salaire minimum, qui comptait 171 employés en 1941, en compte 205 en 1943. Elle émet 8 nouvelles ordonnances en 1941-42 et 1942-43. En 1942, elle abaisse le taux des prélèvements de $1 / 5$ à $1 / 6$ de $1 \%$. Le surplus accumulé au 31 mars 1942 est de \$530,000. Nous entendons pour la première fois parler en 1943 du Service forestier, ou «Service de l'exploitation forestière ». Ce service a son origine dans le mécontentement croissant des travailleurs en forêt jusqu'en 1933, alors qu'éclate une grève violente accompagnée de l'intervention de la police. Une enquête révèle qu'un grand nombre d'ouvriers de la forêt ne recevaient même pas $\$ 1$ par jour pour la coupe et le charroyage, et $\$ 1.60$ pour le flottage; les salaires furent immédiatement augmentés, et la rétroactivité fut ordonnée. Une loi suit $(24 \mathrm{Geo}$. V, ch. 22 , du 20 avril 1934), qui crée la «Commission de surveillance et de contrôle des opérations forestières ». Deux ans plus tard cette commission est abolie, et ses attributions sont transférées au Ministère des terres et forêts, puis, en 1940, à la Commission du salaire minimum. Celle-ci agit efficacement dans ce domaine deux ans plus tard par l'émission de l'ordonnance 39, qui réglemente la coupe, le charroyage et le flottage du bois, et englobe 116,472 travailleurs, soit près du tiers de l'ensemble des travailleurs atteints par la loi.

Le service d'inspection compte, en 1943, 62 employés, soit 5 de plus qu'en 1941; il accorde 1,146 permis d'heures supplémentaires, et enregistre 11,300 noms d'enfants âgés de 14 à 16 ans, ainsi que 49,000 fem- 
mes. - Au cours de cette période, 48 employés du placement sur 124 passent à l'assurance-chômage fédérale. Le service a subi une importante transformation à la suite du fonctionnement de la Commission fédérale d'assurance-chômage et de l'ouverture, dans divers centres, de bureaux de placement établis par cet organisme. La loi fédérale de coordination des bureaux de placement a cessé de s'appliquer, de sorte que notre service est désormais entièrement sous le contrôle de la Province; Québec a d'ailleurs été la seule province à maintenir un service séparé de placement. A la suite d'une entente avec le fédéral, le service provincial de placement ferme 9 bureaux au début de 1942, n'en conservant plus que 15 réguliers et quelques temporaires. Ceci explique les chiffres suivants: 148,052 placements en $1941-42$, et 97,735 en 1942-43.

Les pensions aux vieillards et aux aveugles, et les allocations aux mères nécessiteuses occupent 235 fonctionnaires en 1942 (184 en 1941). A 47,450 vieillards vivants au 31 décembre 1942 , la province distribue sa part (1/4) au montant de $\$ 2,317,907$; la pension mensuelle moyenne s'élève à $\$ 17$. A la même date, 2,146 aveugles ont reçu de la commission $\$ 124,760$, représentant la part de la province (1/4). Enfin, 8,459 mères nécessiteuses reçoivent durant l'année $\$ 2,707,291$, ce qui porte l'allocation mensuelle moyenne à \$29.17. — La Commission des accidents du travail, qui emploie au delà de 250 personnes, accuse un surplus de plus d'un million de dollars pour la décennie 1931-1941; dès décembre 1942, le surplus accumulé est doublé; les accidents durant 1942-43 se chiffrent à près de 100,000; au cours de la même période, les dépenses d'administration s'élèvent à près de $\$ 600,000$. La comptabilité du ministère lui-même indique des revenus de $\$ 337,029.35$, et des dépenses de $\$ 6,407,498.65$ pour 1942-43. - Le service de conciliation énumère, pour 1942 et 1943, plus de 200 conflits dans le Québec, et s'occupe de 63 de ces conflits. L'incidence marquée des grèves est un phénomène national, qui exige des remèdes immédiats et durables.

A l'échelle nationale, en effet, la période 1941-43 est marquée par une recrudescence des conflits de travail. Les syndicats, dont les effectifs s'accroissent constamment, se plaignent que la réglementation existante ne réussit pas, dans la pratique, à protéger efficacement le droit d'association et de négociation collective; les patrons, de leur côté, soutiennent qu'il est souvent difficile de déterminer quel syndicat doit être reconnu comme agent négociateur, à cause de la pluralité syndicale. Peu à peu, les autorités fédérales se rendent compte que le mécanisme établi en vertu de la Loi des enquêtes en matière de différends industriels était trop spécifique pour s'adapter sans heurts aux nombreux conflits éclatés dans les industries de guerre, ainsi qu'aux problèmes posés par une main-d'oeuvre toujours plus nombreuse. Le rapport de l'enquête menée par le Conseil national du travail en temps de guerre - rapport McTague, du nom de son président - pour faire le joint de la situation industrielle au pays, indique en 1943 les causes du malaise croissant: le droit de négociation frustré, l'opposition patronale au syndicalisme, la crise 
économique dont les effets se font encore sentir, les rivalités syndicales. Le rapport propose comme remèdes un Code pour les industries de guerre favorisant la convention collective, l'arbitrage obligatoire et la sentence exécutoire, la participation des travailleurs à la marche de l'entreprise et des commissions gouvernementales, les indemnités de vie chère, et le paiement aux femmes d'un salaire égal pour travail égal.

Tous ces faits menèrent à l'adoption des Règlements des relations ouvrières en temps de guerre (C. P. 1003) le 17 février 1944. Cet arrêté fédéral suspendait la Loi des enquêtes en matière de différends industriels, et exigeait que les employeurs négocient de bonne foi avec les représentants des travailleurs. On établit un Conseil des relations ouvrières en temps de guerre, composé d'un nombre égal de représentants des employeurs et des travailleurs, et responsable de l'application des règlements. Ceux-ci autorisaient également le ministre fédéral du Travail à désigner des conciliateurs et des commissions de conciliation chargés d'étudier et de résoudre les différends industriels, et interdisaient les grèves ou contre-grèves pour une période de 14 jours suivant le dépôt entre les mains du ministre du rapport de la commission de conciliation. Les règlements établissaient également des sanctions spécifiques contre les «pratiques déloyales». Ils ne visaient pas les conflits relatifs aux salaires, lesquels tombaient sous le coup de l'ordonnance régissant les salaires en temps de guerre. En vertu de ces règlements, le gouvernement fédéral étendait sa juridiction aux relations du travail - normalement du ressort exclusif des provinces - , afin d'englober l'industrie de guerre, mais sans chercher à réglementer les industries qui n'avaient aucun rapport direct avec la production de guerre. Les principales dispositions contenues dans le C.P. 1003 seront dans la suite incorporées à la Loi sur les relations industrielles et sur les enquêtes visant les différends du travail (1948).

L'arrêté C.P. 1003, inspiré du National Labor Relations Act de 1935 - le Wagner Act des Etats-Unis - qui consacrait le droit de négociation collective et interdisait aux patrons les pratiques déloyales et de mauvaise foi, devait avoir une influence directe sur la législation provinciale de 1944, et sur la Loi des relations ouvrières en particulier. Les législateurs provinciaux avaient, en effet, soigneusement étudié le projet d'arrêté fédéral avant sa sanction, qui devait suivre de deux semaines celle de la Loi des relations ouvrières.

Me Marie-Louis Beaulieu décrit bien l'atmosphère de l'époque, et les circonstances qui ont incité le législateur à agir en 1944:

L'année 1944... est une des plus importantes dans la législation du travail (chez nous). Les années 1941, 1942 et 1943 avaient été témoins de nombreux conflits et de plusieurs grèves: conflits dans l'aluminium et le papier; grève des moulins Price; grève des tramways de Montréal; grève dans les services municipaux et menaces de grèves 
dans l'enseignement de la même ville. Le malaise grandissait. Nos lois étaient insuffisantes, surtout en matière de liberté d'association et de négociation de conventions collectives.

Au printemps (avril) 1943, une commission est nommée par le gouvernement provincial, composée de trois éminents magistrats, les honorables juges A. Prévost, Stuart McDougall et Garon Pratte. A l'automne, elle fait son rapport, maintenant connu sous le nom de Rapport Prévost, une des meilleures études faites jusqu'alors sur les problèmes du travail dans notre province, et qui devait servir de base à la Loi des relations ouvrières et à la Loi des différends entre les services publics et leurs salariés... ${ }^{2}$

ANALYSE DE LA LOI DES RELATIONS OUVRIÈRES

(8 Geo. VI, ch. 30, inséré dans S.R.Q. 1941, ch. 162A)

«La Loi de 1944 sur les relations ouvrières changea radicalement le climat des relations de travail», écrit M. Gérard Tremblay. "En bref, elle établit les principes suivants: reconnaissance réaffirmée et explicitée de la liberté syndicale déjà établie par un texte de 1937 contenu dans la Loi des salaires des ouvriers mais abrogé en 1940; détermination des pratiques déloyales interdites aux employeurs et aux salariés; obligation pour l'employeur de négocier de bonne foi une convention collective si lagent négociateur des salariés établit devant la Commission de relations ouvrières qu'il représente la majorité des salariés d'une unité économique; recours obligatoire à la procédure de conciliation et d'arbitrage au cas d'insuccès des négociations directes; suspension du droit de grève et de lock-out tant que les procédures indiquées n'ont pas été épuisées et qu'un délai de quatorze jours n'a pas été compté après la date de réception de la sentence arbitrale par le ministre du travail ${ }^{3}{ }^{3}$

«La Loi des relations ouvrières, inspirée aussi du N.L.R.A. de 1935 », écrit Me Marie-Louis Beaulieu, «loi qui sert de charte au travail aux Etats-Unis, porte sur le droit d'association, la négociation des conventions collectives, qu'elle rend obligatoire pour l'employeur privé aussi bien que pour l'employeur public, sur la grève et l'arbtirage, et crée la «Commission de relations ouvrières », sorte de tribunal d'ordre administratif ayant juridiction en matière de conflits d'intérêts \. ${ }^{4}$

Ces lignes expriment mieux que nous ne saurions le faire le sens et la portée de la Loi des relations ouvrières, qui a incontestablement con-

(2) Beaulieu, Me Marie-Louis, « Législation du travail et de la sécurité sociale 》, notes de cours donnés à l'Université Laval, pp. 59-60. Voir aussi, du même auteur, les pages 175 et suivantes du volume Les conflits de droit dans les rapports collectifs du travail. Québec: Les Presses Universitaires Laval, 1955.

(3) Tremblay, Gérard, «Conventions collectives et extension juridique », Relations industrielles, vol. VII, nos 1-2, p. 3.

(4) Beaulueu, Marue-Louis, op. cit. 
tribué à l'établissement de la paix industrielle chez nous, ainsi qu'au développement du syndicalisme. En deux mots, l'employeur est tenu de négocier de bonne foi avec le groupement ouvrier de son entreprise ou de son établissement, pourvu qu'il soit accrédité par la Commission; si les négociations sont infructueuses, un conciliateur du ministère du Travail intervient; si la conciliation elle-même n'aboutit pas, on doit avoir recours à un conseil d'arbitrage constitué suivant la Loi des différends ouvriers de Québec; la sentence rendue par ce tribunal n'est pas obligatoire; après un délai donné, l'arrêt de travail peut avoir lieu.

\section{LA LOI DES DIFF'́RENDS ENTRE LES SERVICES PUBLICS ET LEURS SALARIÉS}

La Loi des différends entre les services publics et leurs salariés (8 Geo. VI, ch. 31, inséré dans S.R.Q. 1941, ch. 169) «porte sur les mêmes sujets que la Loi des relations ouvrières, et, en plus, elle interdit la grève dans les services publics; mais, pour rétablir en quelque sorte l'équilibre, elle rend la signature de la convention collective obligatoire pour l'employeur $»^{5}$ C'est une loi complémentaire de la précédente. Elle décrète que tout différend concernant les conditions de travail entre un service public - corporations municipales et scolaires, institutions d'assistance publique, asiles d'aliénés et entreprises d'utilité publique et de transport (sauf les chemins de fer sous la juridiction du Canada), et services du gouvernement de la province (mais à l'égard seulement des fonctionnaires et ouvriers visés par la Loi du service civil) et ses salariés doit être soumis à l'arbitrage, selon la formule prévue dans la Loi des différends ouvriers ou dans la convention collective existante, s'il y a lieu. La sentence arbitrale, unanime ou majoritaire, peut être exécutée sous l'autorité d'un tribunal compétent. Toute grève ou lock-out est interdite en toute circonstance dans les services publics.

\section{LES ANNÉES 1944 ET 1945: ACTIVITÉ ADMINISTRATIVE}

Sur le plan administratif, les années 1944 et 1945 sont des années normales. La Commission permanente du Conseil supérieur du travail tient de nombreuses séances. Divers comités étudient la législation sociale, les allocations familiales, le rapport Prévost, les décrets, les tribunaux du travail, l'apprentissage, la sécurité syndicale et les relations entre la Loi de la convention collective et celle des relations ouvrières, et entre la Loi de la convention collective et celle du salaire minimum. Au cours de ces deux années, les divers comités produisent 11 rapports: sur la législation du travail et de la sécurité sociale dans la Province de Québec; sur les allocations familiales et la Loi de la convention collective; sur la Loi de la convention collective elle-même; sur un projet de loi pour les voyageurs de commerce; sur le rapport entre le salaire minimum et la convention collective; sur les négociations collectives obliga- 
toires et les tribunaux du travail; sur le plan Marsh; sur l'industrie de la chaussure; enfin, sur la Loi des accidents du travail. A la suite de ces rapports, des résolutions et des recommandations sont adressées au ministre du Travail.

Le secrétaire de la Commission de relations ouvrières, dans son premier rapport en 1944, écrit ce qui suit:

«Depuis longtemps déjà, les associations ouvrières, dans leur mémoire annuel de recommandations au ministère provincial du Travail, demandaient une législation pour obliger les employeurs à traiter avec l'Union qui représentait la majorité des salariés à leur emploi. Poussé par l'acuité de certains conflits ouvriers dans la province, mû par les recommandations du rapport Prévost et du mémoire du Conseil supérieur du Travail, le Gouvernement, afin de faciliter les relations entre employeurs et employés et éloigner ainsi de nouvelles causes de différends ouvriers, adoptait, à sa Session de 1944, les Statuts 162A et 169 ». (p. 13)

La Commission, dont les trois membres sont nommés le 23 février 1944, pour voir à l'application de ces deux lois que nous venons d'analyser, accorde en mars seulement 29 certificats de reconnaissance, dont 11 dans l'industrie de la pulpe et du papier. En 1944-45, 923 requêtes en reconnaissance sur 1111 sont agréées, intéressant près de 80,000 travailleurs, surtout dans l'industrie - textile, produits chimiques, cigarettes, chaussure, meuble - et dans les services publics - corporations scolaires surtout.

En mars 1945, 94 décrets sont en vigueur. De 1943 à 1945, l'inspecteur des comités paritaires intervient dans 84 cas de litige. - Au début de 1943, 23 ordonnances de salaire minimum sont en vigueur; une nouvelle s'ajoute - pour les verreries - l'année suivante, et plusieurs modifications sont effectuées. Le surplus accumulé de la Commission à la fin de l'exercice financier 1944-45 se chiffre à $\$ 1,119,000$. - Le Service de conciliation et d'arbitrage est particulièrement actif: une trentaine de différends dans les services publics au cours de ces deux années, 57 tribunaux d'arbitrage, 177 grèves - dont 45 relevant du service provincial, près de 100,000 travailleurs impliqués, qui perdent plus de 500,000 journées de travail. Le gros de la bataille se livre autour de la liberté syndicale et du droit de négociation. Le rapport du chef de service indique qu'un grand nombre de patrons - surtout les commissions scolaires - refusent de négocier collectivement. - Dans le domaine de la sécurité des personnes et de la propriété, rien de neuf à signaler; les chiffres indiquent des progrès constants et une expansion notable des divers services - établissements industriels, commerciaux et publics, machines fixes, électriciens et mécaniciens en tuyauterie. - Dans le domaine de l'assurance et de l'assistance sociales, les vieillards, les aveugles et les mères nécessiteuses continuent à percevoir des pensions et 
allocations dépassant $\$ 30,000,000$ par année; le service de placement trouve du travail à plus de 85,000 personnes en 1944-45, les solliciteurs d'emploi se chiffrant à 94,000. - En 13 ans - depuis 1931, la Commission des accidents du travail a payé en compensations plus de $\$ 36,000,000$, ayant perçu en cotisations la somme considérable de \$67,515,011. - La comptabilité du ministère accuse en $1944-45$ des revenus de $\$ 373,161$ et des dépenses de \$8,705,396.

Notons au passage que c'est en 1944 que fut adoptée la Loi fédérale des allocations familiales ( 8 Geo. VI, ch. 40, amendée en 1949); la même année voyait la création du Ministère fédéral de la santé nationale et du bien-être social.

\section{LA LOI DE L'ATDE À L'APPRENTISSAGE 1945}

La Loi de l'aide à lapprentissage et à la mise en valeur du capital humain (9 Geo. VI, ch. 41), sanctionnée le 24 mai 1945, mérite une étude attentive, tant par l'ampleur, l'urgence et l'importance du problème qu'elle aborde que par la qualtié et l'originalité de ses dispositions.

Ainsi se lit l'introduction de ce texte législatif:

Attendu que l'apprentissage est un des plus importants facteurs d'amélioration du sort des classes laborieuses, de progrès général et de saine économie d'un pays;

Attendu que l'adaptation de nos industries aux conditions de l'aprèsguerre et la protection particulière qu'il $y$ aura alors lieu d'apporter à notre capital humain poseront d'une manière aiguë le problème de l'apprentissage;

Attendu qu'il est du devoir de tous d'aider à l'adaptation et à la réadaptation des accidentés du travail, des blessés de guerre et des infirmes;

Attendu que les groupements d'employeurs et de salariés sont vitalement intéressés dans ces problèmes et que les pouvoirs publics doivent aider à les résoudre;

Attendu qu'une des mesures les plus efficaces pour atteindre ces buts est l'établissement de centres d'apprentissage et la constitution de commissions appropriées pour les administrer...

Ce préambule indique à la fois l'inquiétude et la lucidité du législateur en face du grave problème de la formation professionnelle. L'hon. Antonio Barrette, depuis peu de temps ministre provincial du Travail, se fit le champion de ce projet de loi.

«Il était logique que l'aide à l'apprentissage relevât du Ministère provincial du Travail. En effet, l'apprentissage dans la province est 
réglementé en vertu de la loi de la convention collective par l'intermédiaire des comités paritaires. C'est ainsi que l'âge d'admission, la durée de l'apprentissage, les salaires des apprentis et la proportion entre le nombre des apprentis et des compagnons sont déterminés par les conventions collectives, c'est-à-dire par laction conjointe des syndicats ouvriers et des associations patronales. En d'autres termes, toute question reliée directement ou indirectement à l'apprentissage doit se résoudre dans les cadres de la loi de la convention collective.

« C'est ce que fait la loi d'aide à l'apprentissage.

«En vertu de cette nouvelle loi, un comité paritaire, ou une association ouvrière et une association patronale, peut demander qu'une région soit reconnue comme centre d'apprentissage pour un métier déterminé. Si cette requête est agréée par le Gouvernement, les signataires de celle-ci se constituent en Commission d'apprentissage. La loi confère aux commissions tous les pouvoirs dont elles peuvent avoir besoin pour mener à bonne fin l'apprentissage des métiers qui relèvent de leur juridiction. Une Commission d'apprentissage peut élaborer ses programmes, engager des instructeurs, former des sous-comités pour l'orientation professionnelle et le placement des apprentis, signer des ententes avec les écoles professionnelles, etc. Bref, une Commission d'apprentissage jouit d'une grande autonomie.

«Dans les centres où il existe des écoles techniques ou des écoles d'arts et métiers, il est à prévoir que l'enseignement théorique sera diffusé par ces écoles à la suite d'ententes avec les commissions d'apprentissage. On évitera ainsi la duplication des efforts. Aucune incompatibilité n'est à craindre entre ces Commissions d'apprentissage et les écoles professionnelles. Bien au contraire, les deux se complètent mutuellement.

« La loi d'aide à l'apprentissage était nécessaire pour inciter les syndicats et les employeurs à s'occuper eux-mêmes de l'apprentissage. Si, dans le passé, l'apprentissage n'a pas donné les résultats qu'on était en droit d'attendre, cela s'explique par l'absence des syndicats et des associations patronales dans l'élaboration des systèmes d'apprentissage. Ce temps est fini $\gg .{ }^{6}$

La nouvelle loi donne donc à l'entreprise privée, par l'entremise des comités paritaires et des commissions d'apprentissage, le pouvoir d'établir un système d'apprentissage bien adapté. «Par la facilité qu'elle donne à la profession de s'organiser, la Loi de l'aide à l'apprentissage constitue un pas franc dans le sens de la promotion de la classe ouvrière et de l'organisation professionnelle complète. En effet, les commissions d'apprentissage, issues de cette loi, sont formées de représentants patro-

(6) Bulletin des relations industrielles, vol. I, no 1, 15 septembre 1945, p. 5. 
naux et ouvriers. Ces représentants siègent autour d'une même table, et sont responsables de l'administration des centres d'apprentissage et de la préparation des programmes. Pour qui a étudié les systèmes d'apprentissage dans le monde, cette responsabilité donnée aux représentants de la profession est surprenante et, nulle part ailleurs n'existe une formule aussi riche du point de vue professionnel et démocratique... De plus, la Loi de l'aide à l'apprentissage est peut-être la seule loi qui fasse ressortir avec autant de clarté le point de vue moral dans la préparation des jeunes travailleurs. L'article 17 de la Loi se lit comme suit: « Toute commission d'apprentissage doit prévoir un programme de formation morale des apprentis, qui doit être préparé et appliqué par le conseil de l'instruction publique ». En pratique, cette formation se donne sous forme de cours de sociologie.

«Au point de vue pratique, il y a deux formules différentes selon lesquelles opèrent les commissions d'apprentissage. Dans l'industrie du bâtiment, les commissions d'apprentissage possèdent leurs propres écoles appelées centres d'apprentissage, et voient à l'organisation d'un système d'apprentissage propre à leur industrie... Dans les autres industries, les commissions d'apprentissage travaillent en collaboration avec les écoles de l'Enseignement spécialisé pour la formation des jeunes travailleurs. Dans ce dernier cas, chaque commission a prévu une entente particulière avec une école d'Arts et Métiers ou une école technique, et les conditions de cette entente sont fixées d'après les besoins immédiats de l'apprentissage dans cette région...

«Dans la mise en application de ce système particulier d'apprentissage, le ministère provincial du Travail collabore très étroitement avec les représentants du capital et du travail. Au point de vue financier, il accorde aux commissions qui opèrent leurs propres centres d'apprentissage des octrois annuels substantiels pour les aider à boucler leur budget... De plus, le ministère du Travail met à la disposition des commissions d'apprentissage un Service provincial d'aide à l'apprentissage, dont les officiers agissent comme agents de liaison entre les différents organismes appelés à collaborer ensemble pour le succès de l'apprentissage. Ce Service a un rôle informatif, consultatif et représentatif. Il tend également à doter les commissions d'apprentissage d'une statistique sûre et susceptible de les aider à réglementer d'une façon rationnelle l'apprentissage des métiers $»^{7}$

Nous terminons sur ces propos l'étude d'une période particulièrement difficile, et fort riche en événements d'ordre législatif et administratif. La guerre vient de se terminer - en mai en Europe, en août avec le Japon; - elle a contribué à accroître démesurément le potentiel industriel du pays; elle a déplacé du côté fédéral le centre d'autorité en matières de conflits de travail, de salaires; elle a mis fin à la crise, multipliant le nombre des travailleurs - des femmes surtout - sur le marché 
du travail; elle a vu un accroissement marqué des effectifs syndicaux; enfin, elle a fait progresser notre législation du travail. Parallèlement, les divers services de notre Ministère du travail se sont étendus et perfectionnés; la collaboration avec de fédéral a été plus poussée.

\section{RECHERCHES SOCIOGRAPHIQUES}

Revue publiée par le Département de sociologie et d'anthropologie de I'Université Laval, Québec, Canada.

Direction: Jean-C. FALARDEAU et Fernand DUMONT

Secrétaire de la rédaction: Yves MARTIN

Sommaire du no 1, volume IV, janvier-avril 1963

M.-Adílard TREMBLAY et GÉRALd FORTIN: Enquête sur les conditions de vie de la famille canadienne-française: lunivers des besoins.

Fernand OUELLET: Nationalisme canadien-français et lä̈cisme au XIXe siecle.

Gérald F. McGUIGAN: La concession des terres dans les Cantons de l'Est du Bas-Canada (1763-1809).

ANDRÉ RENAUD, o.m.i.: Communautés ethniques et collectivités indiennes au Canada.

iStuations de la recherche - Comptes rendus - Revue des revues Abennement (un an, trols numéros): Canada: \$4.00: autres pays: \$5.50. - Administration ot abennementa: Les Presses de l'Université Laval, Québec, Canada. - Direction of rédaction: Département do soctologie ot d'anthropologie, Faculté des Sciences sociales. Universitb Laval, Québec, Canade.

(7) Théruen, Chardes-E., statisticien et publiciste du Service de l'aide à l'apprentissage, « Aide à l'apprentissage \, Relations industrielles, vol. 7, no 4, septembre 1952, pp. 287-293. 